\title{
Effects of Stack Position on the Optimum Performance of a Thermo-acoustics Refrigeration System using ABS (Acrylonitrile Butadiene Styrene) Stack Material
}

\author{
Dewi Permata Sari \\ Acoustic Laboratory, Faculty of Industrial Technology, Institut Teknologi Bandung, Jl. Ganesha no.10 \\ Bandung, 40132, Indonesia \\ E-mail:dewips133@gmail.com \\ Ida Bagus Ardhana Putra \& Wisnu Hendradjit \\ Faculty of Industrial Technology, Institut Teknologi Bandung, Jl. Ganesha no.10 \\ Bandung, 40132, Indonesia
}

\begin{abstract}
In this paper, the optimum thermo-acoustic stack position was investigated. Two types of parallel-plate stacks made of celluloid negative film and ABS materials were selected and investigation was carried out over three working frequencies. The ABS stack revealed that the farther of the stack position from the sound source, the higher temperature drop was achieved. The simulation in which only acoustic pressure gradient along the stack was considered, disclosed that a more consistent trend could be expected than that revealed in the experimental study.
\end{abstract}

Keywords: thermoacoustics, parallel-plate stacks, temperature instability, ABS, COMSOL Multiphysics 4.2

\section{Introduction}

Thermo-acoustic effect was previously revealed by L. P. Rijke in 1859 using a simple open glass tube from which sound was created when a hot gauze positioned in the tube cooled down slowly. Later Rijke also produced the same effect when the gauze, made of resistance wire, was continuously supplied by electrical current. With the heat being continuously delivered by the gauze into the air column, the sound is also continuous.

Lord Raleygh, 1887, in his book, the Theory of Sound, explained that oscillations of air column in a Rijke like tube due to heat from iron gauzes stimulated the sound.

The reverse Rijke-effect that later well known as thermo-acoustic effect was first discovered by Johannes Bosscha and subsequently investigated by German physicist Peter Theophil Rieß.

In its development, the interplay between density variation and heat injection due to resonance of a standing-wave in gas medium is capitalized to create temperature differences in two different regions. This is called a standing-wave thermo-acoustic engine, which typically has a thermo-acoustic element called the "stack", a solid component with pores that allow the operating gas fluid to oscillate while in contact with the solid walls. Beside thermal penetration depth, $=\sqrt{2 k / \omega}$, the performance of a standing-wave thermo-acoustic device is also influenced by other factors, such as type, material and optimum distance of the stack from the sound source.

In designing a thermo-acoustic device, the difference between theoretical and real optimum stack positions usually exists. It causes inaccurate prediction of the overall performance of the device. In this paper the optimum stack position and its correlation with other design parameters will be analyzed using an experimental set up. 


\section{Standing Wave Phenomena in The Thermo- Acoustic Model}

\subsection{A Standing Wave in A Resonator Tube.}

If a sound source is created in a resonator tube with a closed end (see Fig. 1), standing waves will be created along the resonator tube.

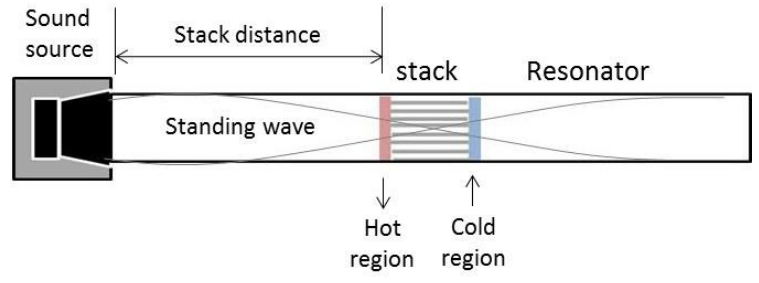

Fig. 1. Schematic set up of a standing wave thermo-acoustic device.

The continuity equation of the sound wave can be described as follows,

$\frac{\partial \rho}{\partial t}+\frac{1}{S} \frac{\partial}{\partial x}(S \rho u)=0$

where $x$ and $S(x)$ are length and surface area of the resonator tube respectively, while $\rho$ and $u$ are air density and particle velocity. The Eq. (1) describes 1dimensional mass flow that assumed to have a constant value. The momentum can therefore be described as follows,

$\frac{\partial}{\partial t}(\rho u)+\frac{1}{S} \frac{\partial}{\partial x}\left(S \rho u^{2}\right)+\frac{\partial p}{\partial x}=-\rho D(u)$

where $p$ is the acoustic pressure and $D(u)$ is the momentum transfer between air and solid surface.

By assuming that the air medium as an ideal gas, the energy equation can therefore be defined as follows, $\frac{\partial}{\partial t}\left(\frac{1}{\gamma-1} p+\frac{1}{2} \rho u^{2}\right)+\frac{1}{S} \frac{\partial}{\partial x}\left[u S\left(\frac{\gamma}{\gamma-1} p+\frac{1}{2} \rho u^{2}\right)\right]=$ $\rho c_{p}\left[\mathrm{H}\left(T_{w}-T\right)-\frac{d T_{w}}{d x} \mathbb{Q}^{\urcorner}\right.$

where $T$ and $T w$ are air temperature and solid wall temperature respectively, while $H$ and $Q$ are derivatives of $D$.

At the closed end of the resonator tube, the particle velocity $u=0$ and $\frac{\partial p}{\partial x}=0$, therefore Eq. (1) and (3) can be combined, as follows,

$$
\begin{aligned}
& \frac{\partial \rho}{\partial t}+\rho \frac{\partial u}{\partial x}=0 \\
& \frac{\partial p}{\partial t}+\gamma p \frac{\partial u}{\partial x}=(\gamma-1) \rho c_{p} \mathrm{H}\left(T_{w}-T\right)
\end{aligned}
$$

Eq. (4) represents standing waves created along the resonator tube, whereas Eq. (5) shows the relationship between the acoustic pressure difference and the air temperature difference as a result of the medium oscillations. The two equations, therefore indicate that the standing waves created along the resonator tube, including stack region, will generate thermo-acoustic effect at both ends of the stack.

On the other hand, the relationship between pressure $(p)$ and temperature $(T)$ of the medium is also indicated by an equation of ideal gas (see Eq. 6) in which the pressure changes of the medium represent the acoustic pressure of the standing waves.

$P V=n R T$

This indicates that characteristics of the medium will also influence thermo-acoustic performance of the resonator tube.

In a standing wave thermo-acoustic system, there are three different kinds of sound wave may occur along the resonator tube.

(i) Standing waves where the pressure vector is perpendicular to the velocity vector (see Fig. 2). The optimum temperature variations will occur only at both ends of the tube resonator.

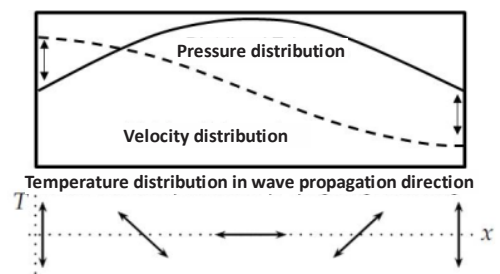

Fig. 2. Pressure, Velocity and Temperature distributions due to standing wave occurrence in a resonator tube.

(ii) Traveling wave occurs along the stack gap/slit. Fig. 3 shows that the velocity vector is parallel to the pressure vector in which adiabatic condition will occur along the stack gap therefore no temperature variation will be created in the stack.

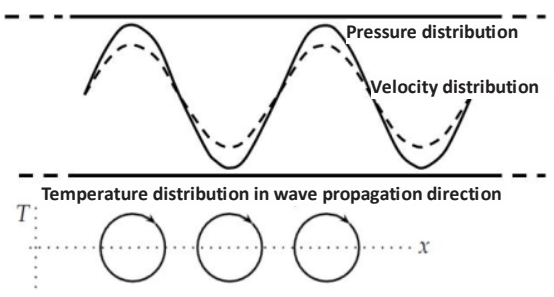

Fig. 3. Pressure, Velocity and Temperature distributions due to traveling wave occurrence in a resonator tube. 
(iii) The interference of standing wave and travelling wave occurs when the stack is positioned in the resonator tube. That causes the changes of temperature distribution along the tube, also the cooling effect formed on area near the stack (see Fig. 4).

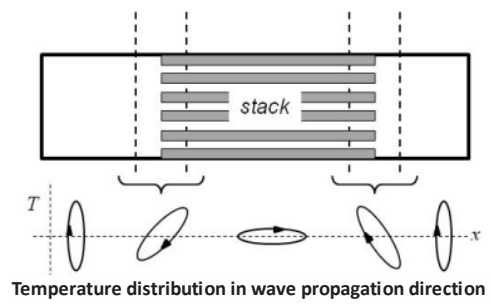

Fig. 4. Pressure, Velocity and Temperature distributions due to interference between standing and traveling wave occurrence in a resonator tube with a stack.

The particle or gas parcel oscillation caused by sound source (loudspeaker) creates the refrigeration effect in which the air parcel temperature on one side of the stack will reduce and increase on the opposite side.

The maximum temperature difference between the two sides of the stack will be achieved when the related pressure difference of the acoustic waves also reaches its optimum value. Fig. 5 illustrates variation of stack position in the resonator tube.
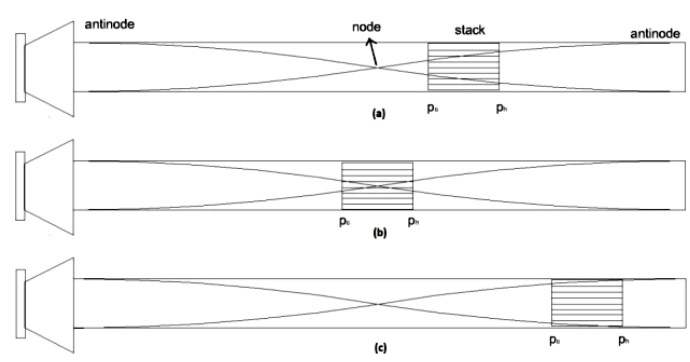

Fig. 5. Variations of the stack position in relation with node and anti-node locations of the standing wave.

Figure 5(a) shows the stack optimum position, while figures 5(b) and 5(c) indicate the stack positions at node and anti-node of the standing wave in which the pressure difference will be zero hence no temperature difference between the two sides of the stack will be produced.

\section{Thermoacoustic System Design}

\subsection{Thermo-acoustic refrigeration cycle}

Pressure changes due to oscillating gas particles during the propagation of sound waves inside the resonator will be followed by temperature changes as shown in Fig. 6 . In one full oscillation cycle, temperature and pressure changes of the air particles signify a refrigeration cycle. Heat absorption and heat release can be identified as $Q_{c}$ and $Q_{h}$, while region $W$ shows the required work during the refrigeration cycle. Fig. 6 also explains that the thermo-acoustic phenomenon is created by the phase shift between acoustic pressure and air parcel temperature in the area around the stack surface.

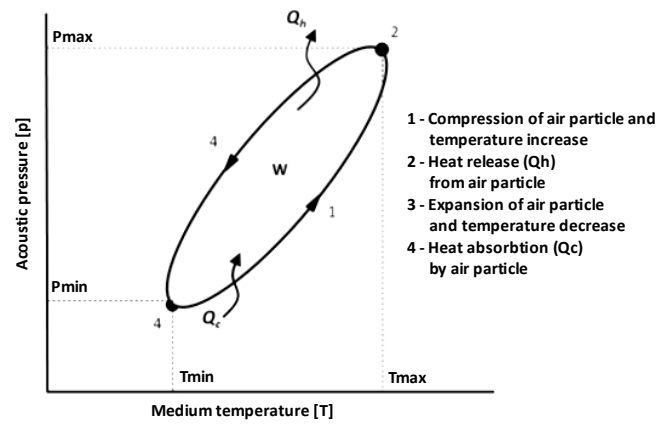

Fig. 6. Pressure and temperature changes during oscillation of gas particle

\subsection{Resonator}

The experiment uses a simple cylindrical resonator with a length $L=\lambda / 4$ that satisfies the following equation:

$$
f=\frac{n v}{4 L}
$$

where $f$ is the selected working frequency and $\mathrm{n}=$ $1,3,5, \ldots$

\subsection{Stack}

The stack as the sole component of thermal energy harvester should be made from a material with low thermal conductivity for preventing heat transfer from the hot to the cold side of the stack ${ }^{1}$.

Based on Rott mathematical equations, thermoacoustic performance is associated proportionally with $\operatorname{Im}(\mathrm{fk})$, or imaginary value of the Rott function ${ }^{1}$. From Fig. 7, the thermo-acoustic optimum performance will 
be generated if the gap width of the stack meets the following equation:

$$
\frac{r_{h}}{\delta_{k}}=\frac{y_{o}}{\delta_{k}}=1,1
$$

where $2 y_{0}$ is the distance between stack layers. The optimum width of stack gap is calculated using the following equation:

$$
2 y_{o}=2,2 \delta_{k}
$$

However, due to difficulties to make stack gap precisely to fit Eq. (9), the gap tolerance stack is defined within the allowable values as follows:

$$
2 \delta_{k}<2 y_{0}<4 \delta_{k}
$$

where $\delta_{k}$ is the thermal penetration depth or the length of heat diffusion occurred during one acoustic period, and can be calculated by the following equation :

$$
\delta_{\kappa}=\sqrt{\frac{\kappa}{\pi f \rho c_{p}}}
$$

The optimum stack position is calculated by:

$$
x=\frac{\lambda}{20}
$$

where $x$ calculated from the closed end of the resonator tube.

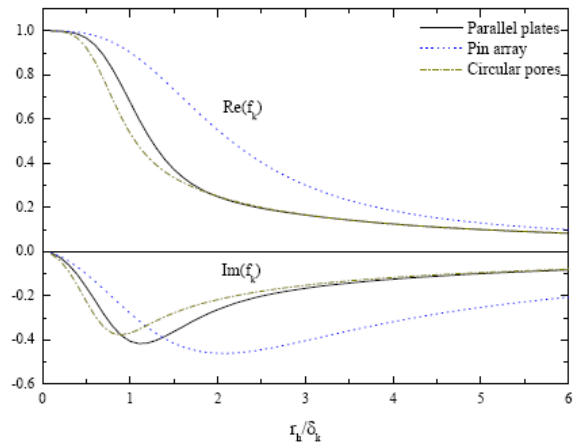

Fig. 7. Real and imaginary values of Rott mathematical equation with $f_{k}$ is a function of ratio of hydraulic radius and thermal penetration depth.

\subsection{The Working Fluid}

The fluid inside the resonator tube functions as a working fluid therefore the performance of the thermoacoustic refrigeration system will depend on thermal conductivity, viscosity, and diffusivity of the fluid. Diffusivity can be described by a thermal penetration depth, while the viscosity is described by the thermal viscosity depth. Thermal viscosity depth is calculated using the following equation:

$$
\delta_{v}=\sqrt{\frac{\eta}{\pi \rho f}}
$$

while diffusivity and viscosity parameters can be expressed in Prandtl number using the following equation:

$$
\sigma=\left(\frac{\delta_{v}}{\delta_{k}}\right)^{2}
$$

\section{Thermo-acoustic Instability}

Thermo-acoustic instability can be characterized by the deviation between the experimental results the theoretical calculations. The main reasons of the instability may be identified as streaming mass, turbulence, curling, transition effects, higher order harmonics, as well as the shock wave ${ }^{2}$.

Streaming is the mass flow that occurs together with the acoustic wave inside the resonator. Panhuis states that streaming will cause degradation of the thermoacoustic performance ${ }^{2}$.

Turbulence arises when the working fluid has a high Reynolds number and the fluid flow in the resonator is no longer laminar. However until now a systematic analysis of the effects of turbulence flow in a thermoacoustic system has not been clearly explained ${ }^{2}$.

The reflection of gas particle at the end of the stack and collisions between gas particles can cause a change in the direction of the particle motion vectors. As a result the vector direction of the acoustic pressure might differ with the heat flow from which the curling effect will appear (see Fig. 8). Fig. 8(a) shows the ideal condition in which the pressure vector is in the same direction as the velocity vector. While Fig. 8(b) shows the condition in which the vector direction of the acoustic pressure is not at the same direction with the velocity vector.

Panhuis research also indicates waveform changes caused by high-order harmonics that will decrease the thermoacoustic performance due to the existence of shock waves. However, more detailed analysis of such phenomenon has not been supported by adequate literatures ${ }^{2}$. 


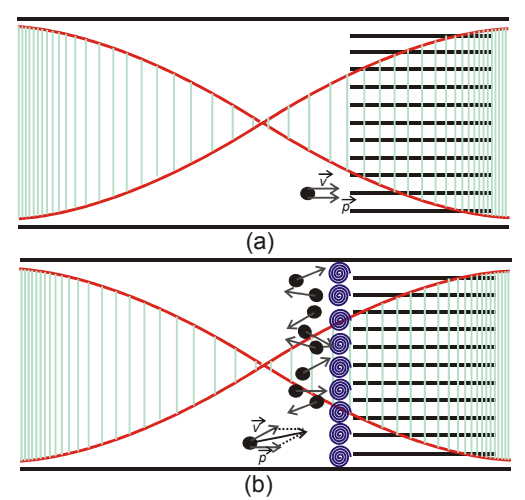

Fig. 8. Curling in front of stack.

In a real condition, some of the instability sources such as streaming, turbulence, high-order harmonics, and the shock wave can be ignored. Streaming and turbulence for example, can be disregarded since the propagation of sound waves in a closed tube does not cause the mass air flow. While the high-order harmonic and shock waves can be discounted because they are not occurring within the system. Therefore the cause of instability that needs to be considered is the curling effect arising in front of the stack due to the reflection of air particles.

\section{Experimental Set Up}

In this study, the selected operating frequency is $150 \mathrm{~Hz}$ which is subsequently used as the main reference for designing other components of the experimental apparatus such as resonators and stack gap. Based on Eq. (7), the required length of the resonator is equal to $62 \mathrm{~cm}$. The type of stack used was parallel plate type with two different types of materials, ABS (Acrylonitrile Butadiene Styrene) and celluloid film negatives (see Fig. 9). From Eq. (11) and (9), the optimum stack gap and the thermal penetration depth of $0.22 \mathrm{~mm}$ and $0.48 \mathrm{~mm}$ are calculated respectively. However, due to difficulties in fabrication, the stack width gap of $0.65 \mathrm{~mm}$ is selected. This value still satisfies Eq. (10). Air at ambient temperature is used as the working fluid. A compression driver (CD) loudspeaker is assembled to construct a sound source from which plane wave radiation will be created along the resonator tube.

The thermo-acoustic performance testing is carried out by measuring the change of air temperatures at both sides of the stack by placing two DS18B20 temperature sensors. Other working frequencies of $170 \mathrm{~Hz}$ and 200 $\mathrm{Hz}$ are also selected to provide adequate data for more comprehensive analysis. A number of stack positions is chosen along the resonator tube, i.e. every $1 \mathrm{~cm}$ within the distance range of $46 \mathrm{~cm}$ up to $56 \mathrm{~cm}$ from the source. The schematic arrangement and the experimental apparatus in the laboratory can be seen in Figure 11 and Figure 12 respectively.

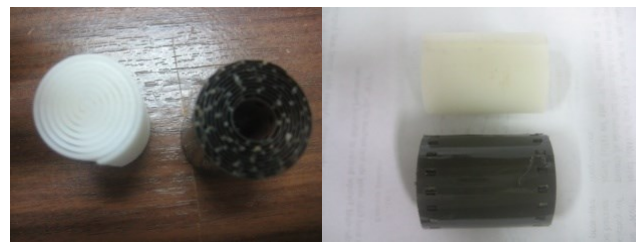

Fig. 9. ABS and celluloid negative film stack.

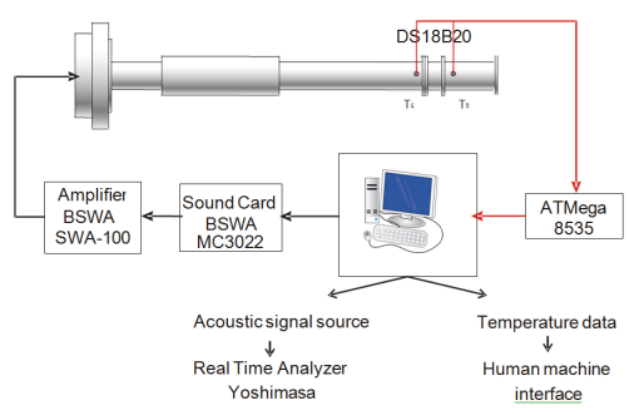

Fig. 10. Schematic arrangement of the experimental measurement.

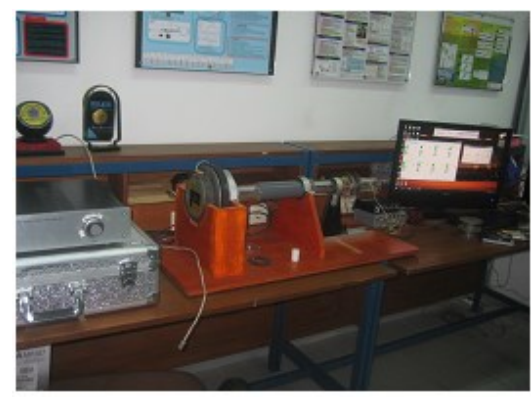

Fig. 11. Thermoacoustic experimental apparatus.

\section{Modelling Using COMSOL Multiphysics 4.2}

In this study, the thermo-acoustic refrigeration system is modeled using COMSOL Multiphysics 4.2 with appropriate definitions of sound hard boundary (wall), plane wave radiation boundary, and thermo-acoustic boundary. 
Other considerations that are employed into the model are as follows:

- The one unit length in the simulation is measured equal to $1 \mathrm{~mm}$ in the experimental condition.

- The tube wall is considered as a sound hard wall

- The stack is a parallel plate type

- The material type of the stack is ignored and considered as a sound hard wall

- The working fluid is air with thermodynamic properties at its ambient temperature as shown in Table 1.

- The loudspeakers placed at one end of the tube is considered to radiate plane waves

- The acoustic pressure in the model is considered equal to the acoustic pressure in the experiment

- The thermodynamic process that may occur inside the stack is ignored, including the thermal contact between air particles with walls of the stack.

The FEM for the simulation model is composed by extremely fine mesh with the smallest element size of $0.0124 \mathrm{~mm}$ and 2099 number of elements within the total simulated area of $7522 \mathrm{~mm}^{2}$. The stack position is shifted every $2 \mathrm{~cm}$, within the distance range of $46 \mathrm{~cm}$ to $56 \mathrm{~cm}$ from the sound source. Beside the working frequency of $150 \mathrm{~Hz}$, the system performance is also analyzed at the center frequencies of $170 \mathrm{~Hz}$ and 200 $\mathrm{Hz}$.

Table 1. Air properties used in simulation.

\begin{tabular}{ll}
\hline Property & Value \\
\hline $\boldsymbol{\mu}$ & $1,82 \times 10-5 \mathrm{~Pa} . \mathrm{s}$ \\
$\boldsymbol{\rho}$ & $1,205 \mathrm{~kg} / \mathrm{m} 3$ \\
$\boldsymbol{\mu B}$ & $1,82 \times 10-5 \mathrm{~Pa} . \mathrm{s}$ \\
$\mathbf{C p}$ & $1,005 \mathrm{~kJ} / \mathrm{kg} . \mathrm{K}$ \\
$\boldsymbol{\kappa}$ & $2,57 \times 10-\mathrm{W} /(\mathrm{m} . \mathrm{K})$ \\
$\mathbf{R}$ & $287,04 \mathrm{~J} /(\mathrm{kg} . \mathrm{K})$ \\
\hline
\end{tabular}

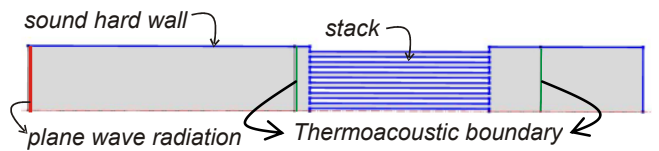

Fig. 12. Thermoacoustic modeling using COMSOL Multiphysics 4.2.

\section{Result and Discussion}

\subsection{Standing wave analysis}

At the working frequency of $150 \mathrm{~Hz}$, the acoustic pressure along resonator is found almost consistently increasing beyond the $6 \mathrm{~cm}$ distance from the sound source up to the mid area of the stack (see Fig. 13). A fluctuating acoustic pressure can be identified up to 6 $\mathrm{cm}$ in front of the loudspeaker due to near field phenomena in which destructive and constructive interference of sound waves can be expected. Consequently, the acoustic pressure measurement in this area in neglected.

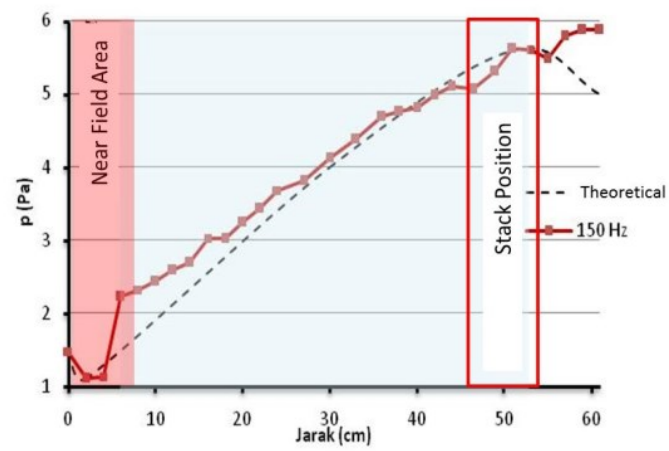

Fig. 13. Acoustic pressure along resonator in freq of $150 \mathrm{~Hz}$.

The near field phenomenon for other frequency of interest $(170 \mathrm{~Hz}$ and $200 \mathrm{~Hz})$ occurs within $8 \mathrm{~cm}$ from the source, as shown in Fig. 14 and Fig. 15.

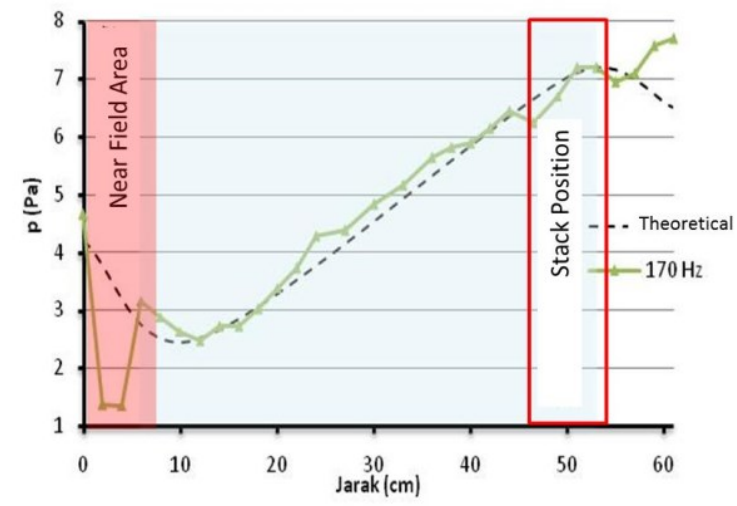

Fig. 14. Acoustic pressure along resonator in freq of $170 \mathrm{~Hz}$.

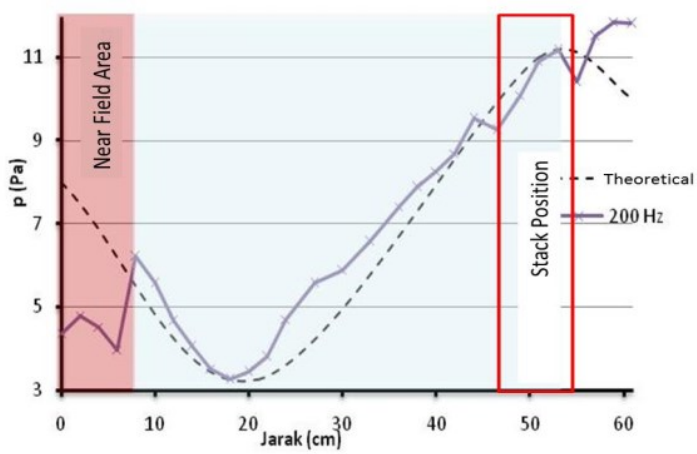


Fig. 15. Acoustic pressure along resonator in freq of $200 \mathrm{~Hz}$.

In accordance with plane wave characteristic in a resonator tube, the ideal condition can be achieved if the anti-nodes are located at closed end of the tube and the source positions. However, the measurement results show that the anti-nodes of the standing wave is not exactly positioned at the closed-end of the tube, but at distances of $6 \mathrm{~cm}$ and $8 \mathrm{~cm}$ from the closed end of the tube. Therefore, the measurement is considered valid at positions after the near-field area up to the anti-node position or at the locations between $46 \mathrm{~cm}$ and $55 \mathrm{~cm}$ from the source. Within this range significant pressure differences between the two sides of the stack can be achieved.

\subsection{Experimental and simulation result}

Table 2. Temperature decrease in cold compartment using ABS stack.

\begin{tabular}{|c|r|r|r|r|r|r|r|r|r|r|}
\hline \multirow{2}{*}{$\begin{array}{c}\text { Freq } \\
(\mathrm{Hz})\end{array}$} & \multicolumn{10}{|c|}{ Stack position from sound source $(\mathrm{cm})$} \\
\cline { 2 - 12 } & 46 & 47 & 48 & 49 & 50 & 51 & 52 & 53 & 54 & 55 \\
\hline 150 & 0,12 & 0 & 0 & 0,12 & 0,25 & 0,25 & 0,25 & 0,38 & 0,25 & 0,25 \\
\hline 170 & 0,25 & 0,37 & 0 & 0,12 & 0,5 & 0,5 & 0,38 & 0,5 & 0,5 & 0,5 \\
\hline 200 & 0,38 & 0,63 & 0,5 & 0,62 & 0,75 & 0,83 & 0,75 & 0,75 & 0,63 & 1 \\
\hline
\end{tabular}

Table 3. Temperature decrease in cold compartment using celluloid negative film stack

\begin{tabular}{|r|r|r|r|r|r|r|r|r|r|r|}
\hline \multirow{2}{*}{$\begin{array}{c}\text { Freq } \\
(\mathrm{Hz})\end{array}$} & \multicolumn{10}{|c|}{ Stack position from sound source $(\mathrm{cm})$} \\
\cline { 2 - 11 } & 46 & 47 & 48 & 49 & 50 & 51 & 52 & 53 & 54 & 55 \\
\hline 150 & 0,87 & 1,25 & 1,5 & 1,25 & 1,5 & 1,37 & 1,25 & 1,13 & 1,5 & 1,25 \\
\hline 170 & 1,38 & 1,75 & 2 & 1,5 & 2 & 2,25 & 2,38 & 2,25 & 2,37 & 2,13 \\
\hline 200 & 2,38 & 2,5 & 2,63 & 2 & 2,38 & 3,83 & 3,87 & 3,75 & 3,88 & 3,25 \\
\hline
\end{tabular}

Table 4. Temperature decrease in cold compartment in simulation.

\begin{tabular}{|r|c|c|c|c|c|c|c|c|c|c|}
\hline \multirow{2}{*}{$\begin{array}{c}\text { Freq } \\
(\mathrm{Hz})\end{array}$} & \multicolumn{10}{|c|}{ Stack position from sound source $(\mathrm{cm})$} \\
\cline { 2 - 11 } & 46 & 47 & 48 & 49 & 50 & 51 & 52 & 53 & 54 & 55 \\
\hline 150 & 0,30 & 0,30 & 0,30 & 0,30 & 0,31 & 0,32 & 0,33 & 0,33 & 0,33 & 0,32 \\
\hline 170 & 0,45 & 0,44 & 0,44 & 0,45 & 0,47 & 0,49 & 0,51 & 0,51 & 0,51 & 0,49 \\
\hline 200 & 0,82 & 0,80 & 0,79 & 0,82 & 0,86 & 0,90 & 0,93 & 0,94 & 0,96 & 0,94 \\
\hline
\end{tabular}

In the experiment using ABS stack, the highest temperature decrease in the cold compartment is $1 \mathrm{C}^{\mathrm{O}}$ with the stack position of $55 \mathrm{~cm}$ and working frequency $200 \mathrm{~Hz}$ (see Table 2). While on the experiment using celluloid stack, the highest temperature decrease in the cold compartment is $0.87 \mathrm{C}^{\mathrm{o}}$ with the stack position of $52 \mathrm{~cm}$ and $55 \mathrm{~cm}$ from the sound source and working frequency $200 \mathrm{~Hz}$ (see Table 3). From the simulation, the highest temperature drop in the cold compartment is $0.96 \mathrm{C}^{\mathrm{o}}$ with the stack position of $54 \mathrm{~cm}$ and working frequency $200 \mathrm{~Hz}$ (see Table 4). The results of experiments using both types of the stack as well as the simulation results indicate that the maximum performance is not achieved at the design frequency of $150 \mathrm{~Hz}$, but at a frequency of $200 \mathrm{~Hz}$.

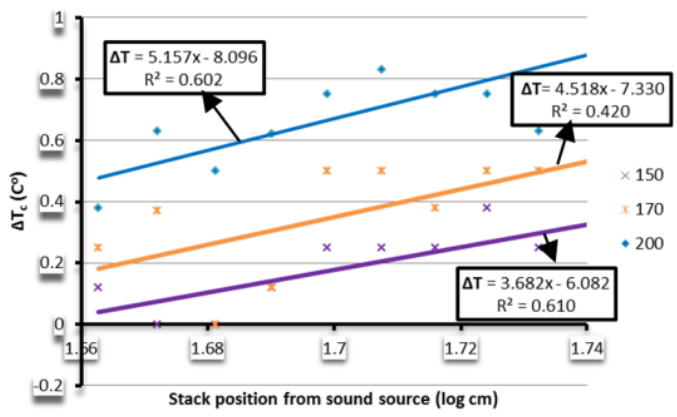

Fig. 16. Linear correlation between temperature decrease on the cold compartment and stack distance using the ABS stack material

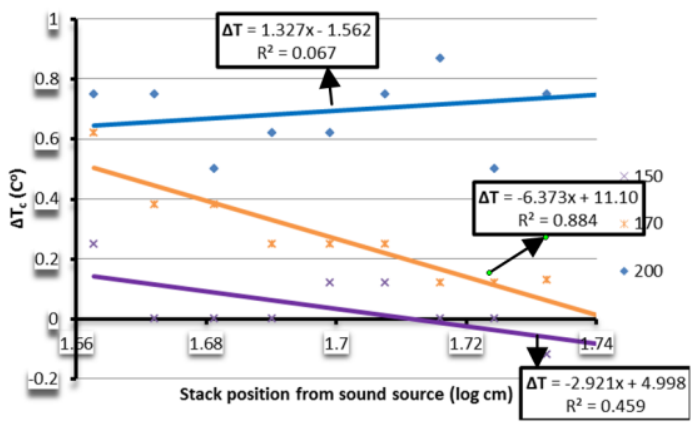

Fig. 17. Linear correlation between temperature decrease on the cold compartment and stack distance using the celluloid stack material

Experiment using ABS stack indicated that temperature gradient in the three working frequency is not much different (see Fig. 16). However, the experiments using negative film stack produce a significant temperature drop only at the working frequency of $200 \mathrm{~Hz}$. While at the working frequency of $150 \mathrm{~Hz}$ or $170 \mathrm{~Hz}$, a negative correlation is obtained (see Fig. 17). Celluloid negative films stack used in the experiment is less robust and has inaccurate gap and misshapen wall that causes gas particle collision on the surface of the stack hence leads to instability phenomenon. The instability made the standing wave inside the resonator cannot be generated perfectly. This has caused inconsistent thermo-acoustic performance 
for a wider frequency range. Therefore more robust and precise stack like ABS provides better performance than the celluloid negative films stack.

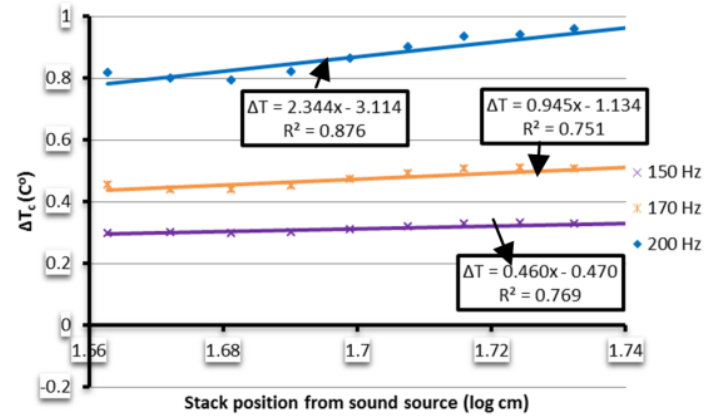

Fig. 18. Linear correlation between temperature decrease on the cold compartment and stack distance derived from the simulation using COMSOL Multiphysics 4.2.

The simulation result shows similar temperature gradient for all three working frequency. However, when it is compared with the experimental results, it can be seen that the simulation provides smaller temperature decrease. In the simulation, only constant pressure gradient is taken into account, while other physical phenomena such as heat backflow through the stack of materials, the effect of thermal contact in the stack plate and instability phenomena are ignored. Therefore, the simulation shows a more consistent result but smaller temperature decrease.

\subsection{Instability phenomenon}

The phenomenon of thermo-acoustic instability can be characterized by the deviation between the measurement and the theoretical results. In theory, the position of the stack is calculated by Eq. (12) considering the distance of $46 \mathrm{~cm}$ from the sound source. However, experimental results showed that the optimum position of the stack is $55 \mathrm{~cm}$ from sound source for the working frequency of $150 \mathrm{~Hz}$. However the best thermo-acoustic performance was obtained at the working frequency of $200 \mathrm{~Hz}$. Table 8 shows the comparison of design parameters and performance between theory, experimental, and simulation.

In the simulation, the instability phenomenon was not taken into account, so that the simulation results show more consistent results. Nevertheless, in this study the phenomenon of instability that arises in experiments is not discussed. In this study, the cause of instability phenomenon that may occur is assumed to be influenced by the curling effect arising on the front surface of the stack.

\section{Summary}

In general, ABS stack provides better performance than the celluloid negative film stack. This is indicated by larger temperature decrease in the cold compartment and more consistent results in various selected working frequencies and stack positions.

Over the three working frequencies, the thermoacoustics system using ABS stack reveals that the farther of the stack position from the sound source, the higher temperature drop will be achieved.

Optimum stack position and working frequency are affected by the instability phenomenon due to the curling effect. In a more robust stack material (ABS) curling effect is smaller than in the celluloid film negatives stack. If the instability can be ignored completely as in the simulation, the temperature decrease will be more consistent for any changes in stack position.

\section{References}

1. Tijani, M. E. H. Loudspeaker-driver Thermo-acoustic Refrigeration (M.S. Thesis, Technische Universiteit Eindhoven, Eindhoven, 2001).

2. Panhuis, P.H.M.W. Mathematical Aspects of Thermoacoustic (Eindhoven University of Technology, Eindhoven, 2009).

3. Karpov, S. and Andrea Prosperettia. A Nonlinear Model of thermoacoustic Devices (Department of Mechanical Engineering, The Johns Hopkins University, Maryland, 2002).

4. Setiawan, I., dkk. Rancang Bangun Piranti Termoakuktik Sebagai Pemompa Kalor (Sigma Vol 10 no.1, Universitas Gajah Mada, Yogyakarta, 2007).

5. Swift, G. What is Thermoacoustics? A Brief Description, with Technical Details and Citations (Condensed Matter and Thermal Physics Group, Los Alamos National Laboratory, Los Alamos, 2004). 